\title{
Towards Accurate, Automatic Segmentation of the Hippocampus and Amygdala from MRI
}

\author{
D. Louis Collins ${ }^{1,2}$ and Jens C. Pruessner ${ }^{1,3}$ \\ ${ }^{1}$ McConnell Brain Imaging Center, Montreal Neurological Institute \\ ${ }^{2}$ Department Biomedical Engineering \\ ${ }^{3}$ Douglas Hospital Research Center, Department of Psychology \\ McGill University, Montreal, Canada \\ \{louis.collins, jens.pruessner\}@mcgill.ca
}

\begin{abstract}
We describe progress towards fully automatic segmentation of the hippocampus (HC) and amygdala (AG) in human subjects from MRI data. Three methods are described and tested with a set of MRIs from 80 young normal controls, using manual labeling of the $\mathrm{HC}$ and $\mathrm{AG}$ as a gold standard. The methods include: 1) our ANIMAL atlas-based method that uses non-linear registration to a pre-labeled non-linear average template (ICBM152). HC and AG labels, defined on the template are mapped through the inverse transformation to segment these structures on the subject's MRI; 2) template-based segmentation, where we select the most similar MRI from the set of 80 labeled datasets to use as a template in the standard ANIMAL segmentation scheme; 3) label fusion methods where we combine segmentations from the ' $n$ ' most similar templates. The label fusion technique yields the best results with median kappas of 0.886 and 0.826 for $\mathrm{HC}$ and $\mathrm{AG}$, respectively.
\end{abstract}

\section{Introduction}

The hippocampus (HC) is a part of the brain located in the medial aspect of the temporal lobe and is part of the limbic system. The HC plays an important role in general and spatial memory in humans and animals, integrating external with internal signals to form a cohesive and unified spatial and temporal orientation of oneself in the environment. HC dysfunction and neurodegeneration has been described in a variety of mental diagnoses, including Alzheimer's disease (AD) [1], Posttraumatic Stress Disorder [2], Major Depression [3], Schizophrenia [4, 5], and epilepsy [6]. The amygdala (AG) lies adjacent to the $\mathrm{HC}$ in the medial temporal lobe, is also part of the limbic system, and is most significantly associated with emotional memory and its regulation. Structural variations of the AG are now being discussed as implicated in mental diagnoses like Schizophrenia and anxiety disorders, and more and more studies now employ volume assessment of the HC and AG from MRI in their protocols [7]. Since $\mathrm{HC}$ and $\mathrm{AG}$ volumes can be important markers of neurodegeneration and can provide a useful outcome measure in clinical trials of new therapies for diseases such as AD, there is significant interest in developing robust, automated methods for segmenting the both the $\mathrm{HC}$ and $\mathrm{AG}$. 
Table 1. Methods survey

\begin{tabular}{llc}
\hline \multicolumn{1}{c}{ Author } & Method summary & HC kappa \\
\hline Hogan JDI;2000,13(2-1):217-8 & landmark + fluid reg. & 0.75 \\
Kelemen TMI,1999.18(10):828-39 & PCA constrained elastic & $0.75(\mathrm{HC}+\mathrm{AG}$ \\
& registration & complex) \\
& Appearance based & 0.800 \\
Klemencic JIST,2004,48(2):166-171 & classification +MRF & $0.79-0.86$ \\
Fischl Neuron,2002. 33(3):341-55. & LDDMM & 0.77 \\
Khan NI, 2008;41(3):735-46. & FreeSurfacer & 0.82 \\
Morey NI, Dec 2008; & FSL/FIRST & 0.79 \\
Morey NI, Dec 2008; & Auto context model + & $0.835-0.859$ \\
Morra NI,2008;43(1):59-68 & adaboost & \\
& Hierarchical EM & $0.808(1)-0.813(\mathrm{r})$ \\
Pohl TMI, 2007;26(9):1201-12 & A priori + graph cuts & $0.852(1)-0.864(\mathrm{r})$ \\
Van der Lijn NI,2008;43(4): 708-20. & b-spline + label fusion & $0.81(1)-0.83(\mathrm{r})$ \\
Heckemann NI,2006;33(1):115-26 & b-spline + label fusion & 0.84 \\
Aljabar NI, 2008;43(2):225-35. & b-spline + label fusion, & 0.88 \\
Gousias NI, 2008;40(2):672-84 & seeding + morphology & 0.87 (young); 0.86 \\
Chupin NI, 2007;34(3):996-1019 & region growing & (AD subj) \\
& Template library + linear & 0.87 (controls); \\
Barnes NI, 2008;40(4):1655-71 & reg + threshold & $0.86(\mathrm{AD} \mathrm{subj)}$ \\
\hline
\end{tabular}

JDI=Journal Digital Imaging, NI=NeuroImage, TMI=IEEE Trans Medical Imaging, JIST=Journal of Imaging Science and Technology.

Manual segmentation of the HC and AG is considered the gold standard for volumetric assessment [8-11]. While it is possible to define a protocol that results in low inter- and intra-rater variability, the procedures remain time-consuming (30-60min per HC) and thus difficult to apply in studies involving large numbers (100s) of subjects. Automatic methods for segmentation require no manual intervention, and thus do not suffer from the problems of inter- and intra-observer variability. Most model-based automatic segmentation methods use one of (i) deformable models [12], (ii) appearance-based models $[13,14]$ or (iii) atlas-based techniques to identify the structure(s) of interest [15-17].

Since a model or template derived from a single individual will be biased in some way for all subjects to be segmented, Barnes et al. developed an efficient 'template library' HC segmentation method where the most similar brain from a group of 55 pre-labeled subjects was selected for the template atlas for registration-based segmentation [18]. Heckemann also developed a segmentation method that took advantage of a library of 30 pre-labeled subjects [19]. Instead of selecting the best template, they used a spline-based non-linear registration technique [20] with each template to generate 30 segmentations for a given subject. Label fusion techniques [21] were then used to combine the segmentations into a single consistent label set for the subject. Since this procedure required considerable computational effort, Aljabar et al. optimized the technique by selecting the ' $n$ ' most appropriate atlases from the library using normalized mutual information [22]. Recently, Chupin et al. reported on an ingenious Markovian model region growing procedure that uses morphometric and topological constraints with anatomical rules to identify $\mathrm{HC}$-specific landmarks 
segment the HC and AG [23, 24]. At present, the work of Barnes [18], Chupin [23] and Gousias [25] yield the best published segmentation results for hippocampus (see Table 1 for a methods survey).

The goal of the current manuscript is to describe our recently developed fully automatic segmentation protocol for the $\mathrm{HC}$ and $\mathrm{AG}$ in human subjects from MRI data. The method combines atlas-based segmentation with a template library and label fusion. The main contributions are threefold: 1) instead of a b-spline technique, we use the publicly available ANIMAL non-linear registration algorithm for the atlasbased segmentation method $[15,26]$; 2) we compare the technique to two other methods (the standard ANIMAL and a Barnes-like template library technique); and 3) when validated with manual labels from 80 subjects, the results are better than previously published automatic techniques.

\section{Methods}

The T1-weighted (T1w) MRI data (sagittal acquisition, 140-160 contiguous $1 \mathrm{~mm}$ thick slices, $\mathrm{TR}=18 \mathrm{~ms}, \mathrm{TE}=10 \mathrm{~ms}$, flip angle $30^{\circ}$, rectangular field of view of $256 \mathrm{~mm} \mathrm{SI}$ and $204 \mathrm{~mm}$ AP) used in this study come from a group of 152 young, neurologically healthy individuals acquired on a 1.5T Philips Gyroscan in the context of the International Consortium for Brain Mapping (ICBM) project [27]. The local Ethics Committee approved the study and informed consent was obtained from all participants. Eighty subjects (from the 152) were selected to limit the number of manually segmented HC and $A G$ and so that the male $(n=39)$ and female $(n=41)$ groups were comparable in age (mean age $25.09 \pm 4.9$ years), handedness and years of education.

For the three procedures described below, the original T1w MRI data were pre-processed. First, each MRI volume was corrected for image intensity nonuniformity using a method that estimates a multiplicative bias field that maximizes the intensity histogram entropy [28]. Next, each dataset was stereotaxically transformed using an affine transformation into the Talairach-like MNI coordinate system [29] and resampled onto a $1 \mathrm{~mm}^{3}$ isotropic grid using a tri-linear kernel.

$\mathrm{HC}$ and $\mathrm{AG}$ and labels were manually defined using the protocol defined in [8] where intra-class reliability coefficients (ICC) were reported of 0.900 and 0.925 for inter- (4 raters) and intra-rater (5 repeats) reliability, respectively, for the $\mathrm{HC}$ and 0.835 and 0.930 (respectively) for the AG in these 80 young normal controls.

\subsection{Segmentation Procedures}

Three segmentation procedures were employed in the experiments presented below. The first is based on the publicly available ANIMAL segmentation method and uses an average template $[15,26]$. The second uses ANIMAL with the best single subject template selected from a template library (like Barnes [18]). The third uses ANIMAL with the best $n$ templates selected from a template library and combines the $n$ segmentations with label fusion. These three methods are described in greater depth below.

The ANIMAL technique with an average template. (ANIMAL) [15, 26] is an atlasbased segmentation method that uses non-linear registration to a pre-labeled average template to achieve segmentation (Fig. 1). The template labels are mapped through 
the inverse of subject-template registration to identify the structures of interest in the subject's MRI. To estimate the required non-linear transformation, the ANIMAL algorithm attempts to match image grey-level intensity features in local neighbourhoods in a hierarchical fashion by maximizing the cross-correlation of intensities between the subject and template images. The procedure begins by estimating the deformations required to match blurred versions of the subject and template data. The result is a dense deformation field, where a displacement vector is stored at each node of the field that best matches the local neighbourhoods. This deformation field is subsampled and used as input to the next iteration of the procedure, where the blurring is reduced and the estimation of the deformation field is refined. Labels, defined on the template, are mapped though the inverse of the recovered transformation to identify the $\mathrm{HC}$ and $\mathrm{AG}$ on the subject. Since the non-linear registration is imperfect, it is possible that CSF voxels might be included in the label set. A simple thresholding rule $(0.4 *$ median intensity of $\mathrm{HC}$ label) is used to eliminate CSF.

The ANIMAL technique with a template library $(A+$ best template). Even though the average template used by ANIMAL represents the average anatomy, it might not be optimal to segment certain individual subjects. We therefore investigated a second method, inspired by the work of Barnes et al. [18]. We used the 80 labeled MRI volumes described above as a template library. In order to achieve segmentation, the best template for a given subject is selected from the remaining 79 templates in the database in a leave-one-out fashion. The best template is selected using normalized mutual information between the subject to be segmented and each of the potential templates. Instead of using only linear transformations like Barnes [18], in our approach the selected template was used with non-linear registration in the ANIMAL segmentation procedure described above. The advantage of this technique is that the template that is most similar to the subject is used, and thus the non-linear deformations (required to match subject-to-template) are minimized, resulting in good segmentation. The computational cost of this method is slightly higher than the ANIMAL method since it requires evaluation of the normalized mutual information metric with each potential template.

The ANIMAL technique with a template library and label fusion $(A+$ fusion). One of the disadvantages of the method described above is that it is possible that the single template selected may not be optimal for segmentation of the given subject and that errors in the non-linear estimation between subject and template may result in errors in segmentation. For these reasons, we consider combining multiple segmentations to minimize errors and maximize consistency between segmentations. To do so, the best $n$ templates for a given subject are selected from the remaining 79 templates in the database using normalized mutual information. Each template is then used to produce an independent segmentation of the subject using the ANIMAL procedure. The result is $n$ different segmentations and the issue becomes how to combine the segmentations to achieve a single consistent labeling of the subject. Following the work of Rofhling et al. [21], Heckemann et al. [19] and Aljabar et al. [22], we use label fusion. At each voxel, a voting strategy is used; the label with the most votes from the $n$ templates is assigned to the voxel. To avoid bias, a random selection is used when two or more labels tie in the voting scheme. 
Before applying the label fusion method, the optimum number of templates $n$ must be determined. To do so, we applied the technique with $n=2 . .20$ and examined the graph to see how K and S change with $n$ (Fig. 2). Since both kappa and similarity increase with the number of labels fused, but appear to plateau after $n=11$, we decided to use $n=11$ for the label fusion procedure.

The computation cost of this method is much greater than the first two methods. Not only is normalized mutual information estimated for each potential template, but $n$ non-linear registrations must be computed to achieve the final segmentation. The main differences between our work and those cited above are in the choice of nonlinear registration scheme (i.e., ANIMAL vs. b-splines) and in the improved results for $\mathrm{HC}$ and $\mathrm{AG}$ presented below.

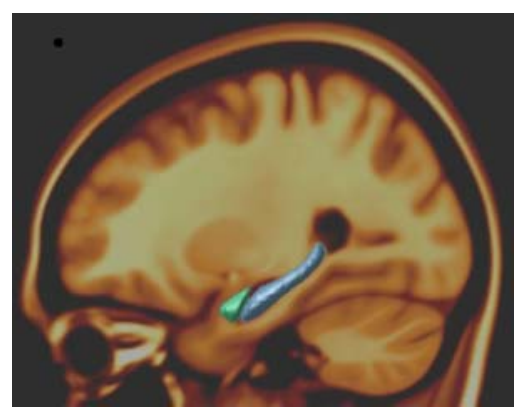

Fig. 1. Sagittal image of ICBM152 nonlinear average template with model $\mathrm{HC}$ (blue) $+\mathrm{AG}($ green $)$

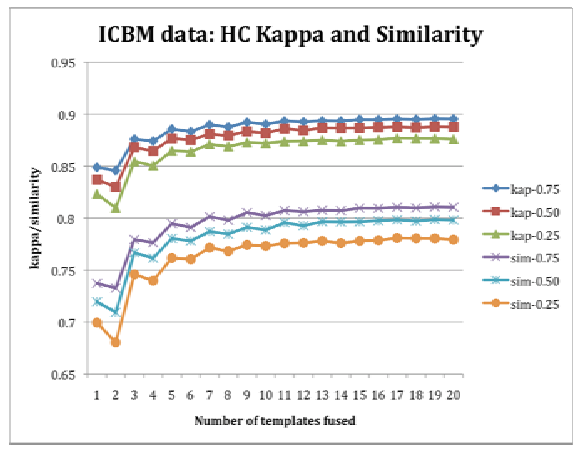

Fig. 2. Kappa (kap-) and Similarity (sim-) plotted (lower quartile $(0.25)$, median $(0.50)$, upper quartile (0.75)) against the number of templates fused

\section{Experiments and Results}

Each of the methods was applied to segment the HC and AG (left and right sides) in the set of $80 \mathrm{MRI}$ volumes. Figure 3 shows the segmentation results on 2 subjects. For each of the methods, the $\mathrm{HC}$ and $\mathrm{AG}$ automatic labels were compared to the gold standard manual labels using Dice's Kappa $[\mathbf{K}=2 *(\mathrm{~V}(\mathrm{M} \cap \mathrm{A})) /(\mathrm{V}(\mathrm{M})+\mathrm{V}(\mathrm{A}))]$; Jaccard Similarity $[\mathbf{S}=(\mathrm{V}(\mathrm{M} \cap \mathrm{A})) /(\mathrm{V}(\mathrm{M} \cup \mathrm{A}))]$ and Normalized volume difference $[\mathbf{D}=2 *$ abs $(\mathrm{V}(\mathrm{M})-\mathrm{V}(\mathrm{A})) /(\mathrm{V}(\mathrm{M})+\mathrm{V}(\mathrm{A}))]$; where $\mathrm{M}$ is the set of manually labeled voxels, $A$ is the set of automatically labeled voxels, $\cap$ is the set intersection operator, where $U$ is the set union operator, $\operatorname{abs}(\bullet)$ is the absolute volume and $\mathrm{V}(\bullet)$ is the volume operator. $\mathbf{K}$ and $\mathbf{S}$ take on a value between 0 and 1.0, with 1.0 indicating perfect agreement. The values of $\mathbf{S}$ are always less than $\mathbf{K}$. D takes on positive values. Values closer to 0.0 are better. The relationships between manual and automatic structure volumes are reported as Pearson's product-moment correlation (r). Tables $2 \& 3$ summarize the quantitative results for $\mathrm{HC}$, and $\mathrm{AG}$, respectively. 
Table 2. Hippocampus segmentation results (median and inter-quartile range)

\begin{tabular}{cccc}
\hline & A + ICBM152 & A + best template & A + fusion \\
\hline $\mathrm{K}$ & $0.864(0.844-0.874)$ & $0.837(0.823-0.849)$ & $\mathbf{0 . 8 8 6}(0.874-0.893)$ \\
$\mathrm{S}$ & $0.761(0.729-0.776)$ & $0.720(0.670-0.738)$ & $\mathbf{0 . 7 9 6}(0.795-0.808)$ \\
$\mathrm{D}$ & $5.5 \%(2.4 \%-10.1 \%)$ & $6.5 \%(3.3 \%-11.1 \%)$ & $\mathbf{4 . 9 \%}(2.7 \%-8.3 \%)$ \\
$\mathrm{r}$ & 0.666 & 0.757 & $\mathbf{0 . 8 3 4}$ \\
\hline
\end{tabular}

Table 3. Amygdala segmentation results (median and inter-quartile range)

\begin{tabular}{cccc}
\hline & A + ICBM152 & A + best template & A + fusion \\
\hline $\mathrm{K}$ & $0.821(0.784-0.842)$ & $0.769(0.736-0.794)$ & $\mathbf{0 . 8 2 6}(0.799-0.856)$ \\
$\mathrm{S}$ & $0.696(0.546-0.727)$ & $0.625(0.582-0.658)$ & $\mathbf{0 . 7 0 3}(0.665-0.748)$ \\
$\mathrm{D}$ & $9.1 \%(4.3 \%-17.4 \%)$ & $12.2 \%(5.6 \%-22.0 \%)$ & $\mathbf{9 . 0 \%}(4.6 \%-15.6 \%)$ \\
$\mathrm{r}$ & 0.605 & 0.405 & $\mathbf{0 . 5 6 6}$ \\
\hline
\end{tabular}

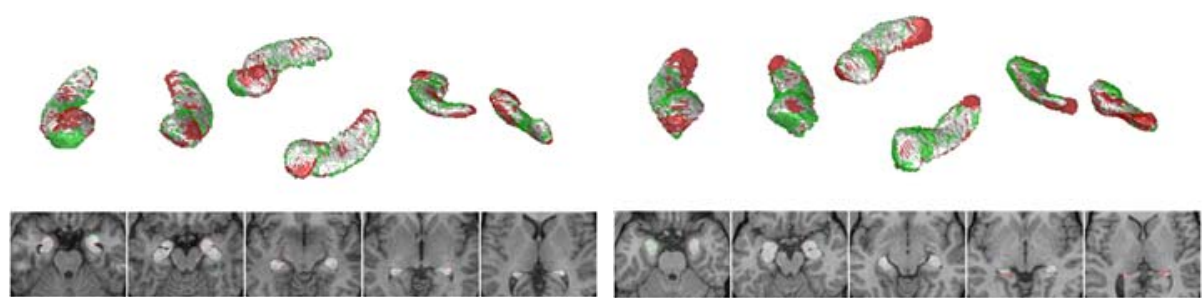

Fig. 3. Example fusion segmentation results for two subjects. White indicates agreement between manual and automatic segmentation, red=false positive and green=false negatives. (note: $\mathrm{HC}$ and $\mathrm{AG}$ are segmented separately, but are presented here together for simplicity) left: $\mathrm{K}_{\mathrm{HC}}=0.89,0.89 ; \mathrm{K}_{\mathrm{AG}}=0.82,0.81$; right: $\mathrm{K}_{\mathrm{HC}}=0.85,0.85 ; \mathrm{K}_{\mathrm{AG}}=0.76,0.82$.

Table 4. Volume results

\begin{tabular}{rcccc}
\hline & $\begin{array}{c}\mathrm{HC} \\
\left(\mathrm{cc}^{3}\right)\end{array}$ & $\begin{array}{c}\text { compared to } \\
\text { manual }\end{array}$ & $\begin{array}{c}\mathrm{AG} \\
\left(\mathrm{cc}^{3}\right)\end{array}$ & $\begin{array}{c}\text { compared to } \\
\text { manual }\end{array}$ \\
\hline Manual & 3.177 & - & 1.121 & - \\
$\mathrm{A}+$ ICBM152 & 3.188 & $\mathrm{p}=0.670$ & 1.067 & $\mathrm{p}<0.001$ \\
$\mathrm{~A}+$ template & 3.226 & $\mathrm{p}=0.038$ & 1.178 & $\mathrm{p}=0.001$ \\
A + fusion & 3.195 & $\mathrm{p}=0.328$ & 1.144 & $\mathrm{p}=0.092$ \\
\hline
\end{tabular}

Table 4 compares the automatic and manual volumes of the segmented structures. The best template method overestimates the true HC volume $(\mathrm{p}=0.038)$, while the other techniques are unbiased. The standard ANIMAL technique underestimates the true AG volume $(\mathrm{p}<0.001)$, while the best template method overestimates its volume $(\mathrm{p}=0.001)$. The fusion technique is unbiased.

\section{Discussion and Conclusion}

We have presented and compared three methods for automatic segmentation of the $\mathrm{HC}$ and AG from MRI of the human brain. The procedures use registration of a 
subject's MRI to a pre-labeled volume to achieve segmentation. The label fusion approach improves on the other two methods because it combines information from multiple sources (i.e., multiple templates) to identify the $\mathrm{HC}$ and AG on the subjects. The fusion of multiple automatic label sets enables the procedure to eliminate inconsistent segmentations that may cause errors in the template library approach where only one template is used.

It is difficult to compare segmentation results between different published methods. The quality of the MRI data, the anatomical definition of the structure, the quality of the manual gold standard, the particular population studied and the different metrics reported make it difficult to compare results. However, with these caveats in mind, we have shown that ANIMAL non-linear registration atlas-based segmentation, combined with a template library and label fusion can achieve high levels of accuracy with a median kappa of 0.887 and similarity of 0.798 for $\mathrm{HC}(0.826$ and 0.796 , respectively for AG). These values are as high or higher than other previously published automatic techniques in the literature (see Table 1).

The label fusion technique was demonstrated to be robust and accurate and yields better results compared to the previous literature in terms of kappa and similarity between manual and automatic labels. In summary, the recent advances in precision in fully automated segmentation techniques will allow application in MRI studies with large number of subjects which previously were impossible because of the large demands in time and human resources.

\section{References}

1. Jack Jr., C.R., Petersen, R.C., Xu, Y., O’Brien, P.C., Smith, G.E., Ivnik, R.J., Boeve, B.F., Tangalos, E.G., Kokmen, E.: Rates of hippocampal atrophy correlate with change in clinical status in aging and AD. Neurology 55, 484-489 (2000)

2. Bremner, J.D., Randall, P., Scott, T.M., Bronen, R.A., Seibyl, J.P., Southwick, S.M., Delaney, R.C., McCarthy, G., Charney, D.S., Innis, R.B.: MRI-based measurement of hippocampal volume in patients with combat-related posttraumatic stress disorder. Am. J. Psychiatry 152, 973-981 (1995)

3. Bremner, J.D., Narayan, M., Anderson, E.R., Staib, L.H., Miller, H.L., Charney, D.S.: Hippocampal volume reduction in major depression. Am. J. Psychiatry 157, 115-118 (2000)

4. Tanskanen, P., Veijola, J.M., Piippo, U.K., Haapea, M., Miettunen, J.A., Pyhtinen, J., Bullmore, E.T., Jones, P.B., Isohanni, M.K.: Hippocampus and amygdala volumes in schizophrenia and other psychoses in the Northern Finland 1966 birth cohort. Schizophr. Res. 75, 283-294 (2005)

5. Buss, C., Lord, C., Wadiwalla, M., Hellhammer, D.H., Lupien, S.J., Meaney, M.J., Pruessner, J.C.: Maternal care modulates the relationship between prenatal risk and hippocampal volume in women but not in men. J. Neurosci. 27, 2592-2595 (2007)

6. Bernasconi, N., Duchesne, S., Janke, A., Collins, D.L., Bernasconi, A.: Voxel-based statistical analysis of grey matter and white matter in patients with unilateral temporal lobe epilepsy. In: Human Brain Mapping Conference (2003)

7. Berretta, S., Pantazopoulos, H., Lange, N.: Neuron numbers and volume of the amygdala in subjects diagnosed with bipolar disorder or schizophrenia. Biol. Psychiatry 62, 884-893 (2007) 
8. Pruessner, J.C., Li, L.M., Serles, W., Pruessner, M., Collins, D.L., Kabani, N., Lupien, S., Evans, A.C.: Volumetry of hippocampus and amygdala with high-resolution MRI and three-dimensional analysis software: minimizing the discrepancies between laboratories. Cereb. Cortex. 10, 433-442 (2000)

9. Matsuoka, Y., Mori, E., Inagaki, M., Kozaki, Y., Nakano, T., Wenner, M., Uchitomi, Y.: Manual tracing guideline for volumetry of hippocampus and amygdala with highresolution MRI. No To Shinkei 55, 690-697 (2003)

10. Bonilha, L., Kobayashi, E., Cendes, F., Li, L.M.: Protocol for volumetric segmentation of medial temporal structures using high-resolution 3-D magnetic resonance imaging. Hum. Brain Mapp. 22, 145-154 (2004)

11. McHugh, T.L., Saykin, A.J., Wishart, H.A., Flashman, L.A., Cleavinger, H.B., Rabin, L.A., Mamourian, A.C., Shen, L.: Hippocampal volume and shape analysis in an older adult population. Clin. Neuropsychol. 21, 130-145 (2007)

12. Ghanei, A., Soltanian-Zadeh, H., Windham, J.P.: Segmentation of the hippocampus from brain MRI using deformable contours. Comput. Med. Imaging Graph 22, 203-216 (1998)

13. Duchesne, S., Pruessner, J., Collins, D.L.: Appearance-based segmentation of medial temporal lobe structures. Neuroimage 17, 515-531 (2002)

14. Kelmencic, J., Pluim, J.P.W., Viergever, M., Schnack, H.G., Valencic, V.: Non-rigid regisgration based on active apearance models for $3 \mathrm{D}$ medical image segmentation. Journal of Imaging Science and technology 48, 166-171 (2004)

15. Collins, D.L., Holmes, C.J., PEters, T.M., Evans, A.C.: Automatic 3D model-based neuroanatomical segmentation. Human Brain Mapping 3, 190-208 (1995)

16. Csernansky, J.G., Joshi, S., Wang, L., Haller, J.W., Gado, M., Miller, J.P., Grenander, U., Miller, M.I.: Hippocampal morphometry in schizophrenia by high dimensional brain mapping. Proc. Natl. Acad. Sci. U S A 95, 11406-11411 (1998)

17. Shen, D., Moffat, S., Resnick, S.M., Davatzikos, C.: Measuring size and shape of the hippocampus in MR images using a deformable shape model. Neuroimage 15, 422-434 (2002)

18. Barnes, J., Foster, J., Boyes, R.G., Pepple, T., Moore, E.K., Schott, J.M., Frost, C., Scahill, R.I., Fox, N.C.: A comparison of methods for the automated calculation of volumes and atrophy rates in the hippocampus. Neuroimage 40, 1655-1671 (2008)

19. Heckemann, R.A., Hajnal, J.V., Aljabar, P., Rueckert, D., Hammers, A.: Automatic anatomical brain MRI segmentation combining label propagation and decision fusion. Neuroimage 33, 115-126 (2006)

20. Rueckert, D., Sonoda, L.I., Hayes, C., Hill, D.L., Leach, M.O., Hawkes, D.J.: Nonrigid registration using free-form deformations: application to breast MR images. IEEE Trans. Med. Imaging 18, 712-721 (1999)

21. Rohlfing, T., Brandt, R., Menzel, R., Maurer Jr., C.R.: Evaluation of atlas selection strategies for atlas-based image segmentation with application to confocal microscopy images of bee brains. Neuroimage 21, 1428-1442 (2004)

22. Aljabar, P., Heckemann, R., Hammers, A., Hajnal, J.V., Rueckert, D.: Classifier selection strategies for label fusion using large atlas databases. Med. Image Comput. Comput. Assist. Interv. Int. Conf. Med. Image. Comput. Comput. Assist. Interv. 10, 523-531 (2007)

23. Chupin, M., Mukuna-Bantumbakulu, A.R., Hasboun, D., Bardinet, E., Baillet, S., Kinkingnehun, S., Lemieux, L., Dubois, B., Garnero, L.: Anatomically constrained region deformation for the automated segmentation of the hippocampus and the amygdala: Method and validation on controls and patients with Alzheimer's disease. Neuroimage 34, 9961019 (2007) 
24. Colliot, O., Chetelat, G., Chupin, M., Desgranges, B., Magnin, B., Benali, H., Dubois, B., Garnero, L., Eustache, F., Lehericy, S.: Discrimination between Alzheimer disease, mild cognitive impairment, and normal aging by using automated segmentation of the hippocampus. Radiology 248, 194-201 (2008)

25. Gousias, I.S., Rueckert, D., Heckemann, R.A., Dyet, L.E., Boardman, J.P., Edwards, A.D., Hammers, A.: Automatic segmentation of brain MRIs of 2-year-olds into 83 regions of interest. Neuroimage 40, 672-684 (2008)

26. Collins, D.L., Evans, A.C.: ANIMAL: validation and applications of nonlinear registration-based segmentation. International Journal of Pattern Recognition and Artificial Intelligence 11, 1271 (1997)

27. Mazziotta, J.C., Toga, A.W., Evans, A., Fox, P., Lancaster, J.: A probabilistic atlas of the human brain: theory and rationale for its development. The International Consortium for Brain Mapping (ICBM). Neuroimage 2, 89-101 (1995)

28. Sled, J.G., Zijdenbos, A.P., Evans, A.C.: A nonparametric method for automatic correction of intensity nonuniformity in MRI data. IEEE Trans. Med. Imaging 17, 87-97 (1998)

29. Collins, D.L., Neelin, P., Peters, T.M., Evans, A.C.: Automatic 3D intersubject registration of MR volumetric data in standardized Talairach space. J. Comput. Assist. Tomogr. 18, 192-205 (1994) 